\title{
The dawn of a new era of targeted therapies for heart failure with preserved ejection fraction (HFpEF)
}

\author{
Kenji Harada $^{1} \cdot$ Kazuomi Kario $^{1}$
}

Received: 12 October 2021 / Accepted: 19 October 2021 / Published online: 24 November 2021

(c) The Japanese Society of Hypertension 2021

Although effective treatments exist for heart failure with a reduced ejection fraction (HFrEF), there is a paucity of treatments with proven benefits for heart failure with a preserved ejection fraction (HFpEF). Detailed results from the phase III EMPEROR-Preserved trial were published in The New England Journal of Medicine on August 27, 2021 [1]. The EMPEROR-Preserved trial was a large, international, double-blind and placebo-controlled trial of empagliflozin, a sodium-glucose cotransporter 2 inhibitor (SGLT2-i), in patients with HFpEF (ejection fraction [EF] $>40 \%$ ). In the trial's HFpEF patients, the SGLT2-i empagliflozin led to a $21 \%$ lower relative risk (hazard ratio [HR] $0.79,95 \%$ confidence interval [CI]: $0.69-0.90$ ) of the composite of cardiovascular death or hospitalization for heart failure, which was related mainly to a $29 \%$ lower risk (HR 0.73 , 95\% CI: $0.61-0.88$ ) of heart failure hospitalization associated with empagliflozin treatment.

In addition, in the trial's evaluation of the influence of the baseline EF according to prespecified subgroups of $41-49 \%, 50-59 \%$ and $\geq 60 \%$ baseline EF on these results, the results demonstrated that the patient's baseline EF did not influence the effect of empagliflozin on the primary endpoint. However, the baseline EF influenced the effect of empagliflozin on the rate of total heart failure hospitalizations, with an attenuated effect in patients with an $\mathrm{EF} \geq$ $60 \%$. This trial can be said to be a landmark study in therapy for $\mathrm{HFpEF}$, for which there has been no effective treatment until now.

In contrast, in the PARAGON-HF trial (Prospective Comparison of ARNI With ARB Global Outcomes in HF With Preserved Ejection Fraction) [2], sacubitril/valsartan (an angiotensin receptor neprilysin inhibitor [ARNI])

Kenji Harada

haradak@jichi.ac.jp

1 Division of Cardiovascular Medicine, Department of Internal Medicine, Jichi Medical University, Tochigi, Japan nonsignificantly lowered $(p=0.058)$ the composite of total heart failure hospitalizations and cardiovascular death compared to valsartan (an angiotensin II receptor blocker [ARB]) in patients with an $\mathrm{EF} \geq 45 \%$. However, interestingly, in the adjusted rate ratio for the primary endpoint by subgroup, the patients with an EF below the 57\% median displayed a significant reduction in the relative risk (HR 0.78, 95\% CI: 0.64-0.95), with a benefit that was consistent with the original study statistical hypothesis. The effectiveness of the two drugs (i.e., sacubitril/valsartan and empagliflozin) for HFmrEF (heart failure with a mid-range ejection fraction) was shown in the PARAGON-HF trial and the EMPEROR trial. Thus, the possibility of a new treatment strategy for HFmrEF was suggested. The results may also indicate that the classification of heart failure using the EF should be reconsidered.

Regarding the treatment of HFpEF, the 2021 European Society of Cardiology (ESC) Heart Failure Guidelines of 2021 note that "to date, no treatment has been shown to convincingly reduce mortality and morbidity in patients with HFpEF" [3]. HFpEF has numerous and complex potential causes, and it; thus, distinct targeted therapies based on the understanding of the mechanisms underlying this condition are required. The 2018 review by Lam et al. describes six mechanisms that translate into potential therapies for HFpEF, beginning with three hemodynamic mechanisms (i.e., left heart congestion/diastolic dysfunction/left atrial hypertension, pulmonary vascular disease/ right ventricular dysfunction, and plasma volume expansion) and working backward to three potential molecular mechanisms (systemic microvascular inflammation, cardiometabolic functional abnormalities, and cellular [titin]/ extracellular [fibrosis] structural abnormalities) [4].

The mechanisms by which an SGLT2-i reduces the number of worsening heart failure events have been unclear. The systematic review by Verma and McMurray summarizes the five following putative mechanisms underlying SGLT2-i-associated cardiovascular benefits from the following aspects: (1) an improvement in ventricular loading 


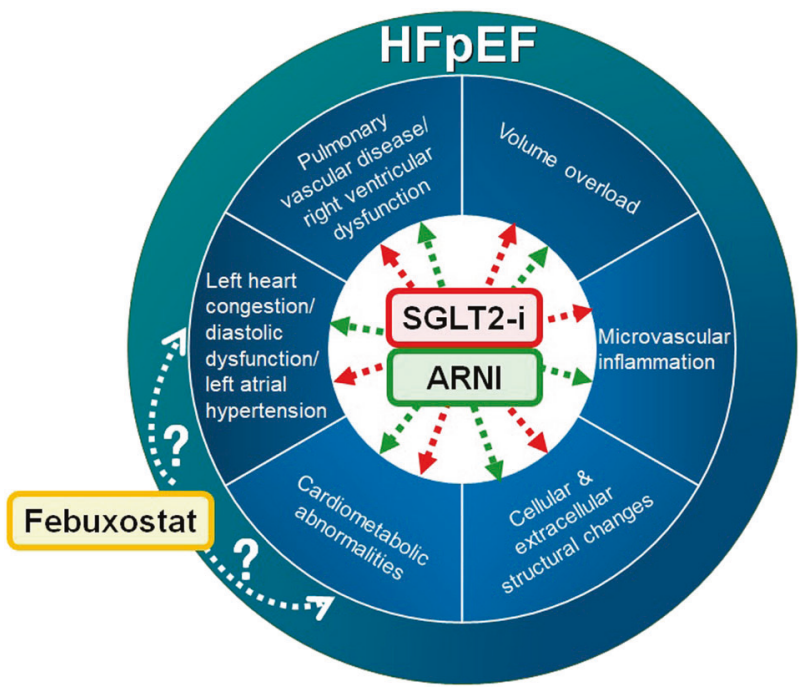

Fig. 1 Recently proposed mechanisms of action of SGLT2-i, ARNI, and febuxostat for the treatment of HFpEF (heart failure with preserved ejection fraction). SGLT2-i sodium-glucose cotransporter 2 inhibitor, ARNI angiotensin receptor neprilysin inhibitor

conditions through a reduction in the preload (secondary to natriuresis, osmotic diuresis) and the afterload (reduction in blood pressure and improved vascular function); (2) an improvement in cardiac metabolism and bioenergetics; (3) an inhibition of myocardial $\mathrm{Na}^{+} / \mathrm{H}^{+}$exchange; (4) reductions in necrosis and cardiac fibrosis; and (5) alterations in adipokines, cytokine production, and epicardial adipose tissue mass [5]. Recent preclinical and clinical research has shown that SGLT2-i treatment provides cardiovascular benefits [6-9]. The early benefits of treatment with an SGLT2-i to prevent clinical deterioration in the EMPEROR-Preserved trial [1] may indicate the possibility of a natriuretic effect, as an acute increase in urinary sodium excretion was caused by the SGLT2-i empagliflozin in a nondiabetic rat model with induced heart failure [10]. Similarly, reports of beneficial action mechanisms in basic research and favorable clinical results for heart failure in ARNI therapy have increased in recent years [11-13]. However, it remains unclear whether similar degrees of cardiovascular benefits from SGLT2-i and ARNI can be translated to HFpEF from the clinical and laboratory outcomes obtained for HFrEF. We illustrate recently summarized possible mechanisms of action of SGLT2-i and ARNI therapy for HFpEF [4] in Fig. 1.

We have read with interest the recently published work in Hypertension Research by Kusunose et al., "Effect of febuxostat on left ventricular diastolic function in patients with asymptomatic hyperuricemia: A sub-analysis of the PRIZE Study" [14]. The purpose of that study was to evaluate the effect of febuxostat on echocardiographic parameters of left ventricular (LV) diastolic function in patients with asymptomatic hyperuricemia as a prespecified endpoint in a subanalysis of the PRIZE study. Kusunose's group reported that additional febuxostat treatment in patients with asymptomatic hyperuricemia for 24 months might have the potential to prevent impaired LV diastolic dysfunction based on echocardiographic Doppler indices such as the $\mathrm{e}^{\prime}$ (early diastolic mitral annular velocity) and the $\mathrm{E} / \mathrm{e}^{\prime}$ (the ratio of early diastolic mitral inflow velocity to $\mathrm{e}^{\prime}$ ). It is interesting that febuxostat treatment led to the protection of LV diastolic function for the pooled cohort of patients with an $\mathrm{EF}>60 \%$. Although the mechanism by which febuxostat protects LV diastolic function for patients with an $\mathrm{EF}>60 \%$ is not yet clear, Kusunose et al. take particular note of the potential direct antioxidant effects of the treatment as reflected in serum uric acid levels as well as its xanthine-oxidase-lowering properties in tissue.

Similarly, in his review Milton Packer described the significance of uric acid as a biomarker of oxidative stress in heart failure [15]. However, he points out that xanthine oxidase inhibitors may increase the risk of cardiovascular death by virtue of an action that masks the beneficial effects of nitric oxide. He also states that SGLT2-i, which acts to mitigate oxidative stress, may simultaneously lead to the lowering of uric acid and cardioprotection because the two actions are related to a shared mechanism. Future elucidation of the mechanisms underlying the association between the action of febuxostat and the amelioration of cardiac function may provide clues for a deeper understanding of not only HFrEF but also HFpEF and the development of novel therapies (Fig. 1). Further studies are needed to clarify the beneficial and long-term effects of febuxostat in patients with advanced heart failure, including HFpEF.

\section{Compliance with ethical standards}

Conflict of interest $\mathrm{KH}$ has no conflicts of interest to disclose. KK reports scholarships from Daiichi Sankyo, Sumitomo Dainippon Pharma, Otsuka Pharmaceuticals, and Takeda Pharmaceuticals and honoraria from Daiichi Sankyo and Novartis Pharma outside the submitted work.

Publisher's note Springer Nature remains neutral with regard to jurisdictional claims in published maps and institutional affiliations.

\section{References}

1. Anker SD, Butler J, Filippatos G, Ferreira JP, Bocchi E, Böhm M, et al. Empagliflozin in heart failure with a preserved ejection fraction. N Engl J Med. 2021. https://doi.org/10.1056/NEJMoa 2107038

2. Solomon SD, McMurray JJV, Anand IS, Ge J, Lam CSP, Maggioni AP, et al. Angiotensin-neprilysin inhibition in heart failure with preserved ejection fraction. N Engl J Med. 2019;381:1609-20.

3. McDonagh TA, Metra M, Adamo M, Gardner RS, Baumbach A, Böhm M, et al. ESC guidelines for the diagnosis and treatment of acute and chronic heart failure. Eur Heart J. 2021;42:3599-726. 
4. Lam CSP, Voors AA, de Boer RA, Solomon SD, van Veldhuisen DJ, Lam CSP, et al. Heart failure with preserved ejection fraction: from mechanisms to therapies. Eur Heart J. 2018;39: 2780-92.

5. Verma S, McMurray JJV. SGLT2 inhibitors and mechanisms of cardiovascular benefit: a state-of-the-art review. Diabetologia. 2018;61:2108-17.

6. Chowdhury B, Luu AZ, Luu VZ, Kabir MG, Pan Y, Teoh H, et al. The SGLT2 inhibitor empagliflozin reduces mortality and prevents progression in experimental pulmonary hypertension. Biochem Biophys Res Commun. 2020;524:50-6.

7. Tanaka H, Soga F, Tatsumi K, Mochizuki Y, Sano H, Toki H, et al. Positive effect of dapagliflozin on left ventricular longitudinal function for type 2 diabetic mellitus patients with chronic heart failure. Cardiovasc Diabetol. 2020;19:6.

8. Hallow KM, Helmlinger G, Greasley PJ, McMurray JJV, Boulton DW. Why do SGLT2 inhibitors reduce heart failure hospitalization? A differential volume regulation hypothesis. Diabetes Obes Metab. 2018;20:479-87.

9. Byrne NJ, Matsumura N, Maayah ZH, Ferdaoussi M, Takahara S, Darwesh AM, et al. Empagliflozin blunts worsening cardiac dysfunction associated with reduced NLRP3 (nucleotide-binding domain-like receptor protein 3) inflammasome activation in heart failure. Circ Heart Fail. 2020;13:e006277 https://doi.org/10.1161/ CIRCHEARTFAILURE.119.006277
10. Borges-Júnior FA, Silva Dos Santos D, Benetti A, Polidoro JZ, Wisnivesky ACT, Crajoinas RO, et al. Empagliflozin inhibits proximal tubule NHE3 activity, preserves GFR, and restores euvolemia in nondiabetic rats with induced heart failure. J Am Soc Nephrol. 2021 https://doi.org/10.1681/ASN.2020071029.

11. Wang Y, Zhou R, Lu C, Chen Q, Xu T, Li D, et al. Effects of the angiotensin-receptor neprilysin inhibitor on cardiac reverse remodeling: meta-analysis. J Am Heart Assoc. 2019;8:e12272 https://doi.org/10.1161/JAHA.119.012272

12. Cunningham JW, Claggett BL, O’Meara E, Prescott MF, Pfeffer MA, Shah SJ, et al. Effect of sacubitril/valsartan on biomarkers of extracellular matrix regulation in patients with HFpEF. J Am Coll Cardiol. 2020;76:503-14.

13. Gori M, Senni M, Claggett B, Liu J, Maggioni AP, Zile M, et al. Integrating high-sensitivity troponin $\mathrm{T}$ and sacubitril/valsartan treatment in HFpEF: The PARAGON-HF Trial. JACC. Heart Fail. 2021;9:627-35.

14. Kusunose K, Yoshida H, Tanaka A, Teragawa H, Akasaki Y, Fukumoto Y, et al. Effect of febuxostat on left ventricular diastolic function in patients with asymptomatic hyperuricemia: a sub analysis of the PRIZE Study. Hypertens Res. 2021; https://doi.org/ 10.1038/s41440-021-00752-9.

15. Packer M, Packer M. Uric acid is a biomarker of oxidative stress in the failing heart: lessons learned from trials with allopurinol and SGLT2 inhibitors. J Card Fail. 2020;26:977-84. 